\title{
Directional Sensitivity, WIMP Detection, and the Galactic Halo
}

\author{
Craig J. Copi ${ }^{1}$, Junseong $\mathrm{Heo}^{2}$ and Lawrence M. Krauss ${ }^{1,3}$ \\ ${ }^{1}$ Department of Physics, ${ }^{3}$ Department of Astronomy \\ Case Western Reserve University, 10900 Euclid Ave., Cleveland OH 44106-7079 \\ ${ }^{2}$ Department of Physics, Yale University, New Haven, CT 06520-8120
}

(June 30, 2018)

\begin{abstract}
Distinguishing the signals due to scattering of WIMP dark matter off of nuclear targets from those due to background noise is a major challenge. The Earth's motion relative to the galactic halo should produce halo-dependent seasonal modulation in the event rate, but it also should produce an angular signal that is both far stronger and less ambiguous. Distinct patterns in the recoil spectrum can reflect the details of the galactic halo. We derive a new formalism to calculate angular event rates, and present the predicted angular signal for a variety of halo models and calculate the number of events needed to distinguish a dark matter signal from an isotropic background.
\end{abstract}

The direct experimental detection of WIMP dark matter would have profound implications for both particle physics and cosmology. Unfortunately, the signature, excess energy deposits due to scattering on nuclei, is not easily distinguishable from various radioactive background signals. A significant number of events might be needed before a definitive observation is believable. Moreover, the recoil spectrum alone is largely independent of many features of the galactic halo distribution, so that our ability to distinguish between galactic halo models will be limited by such observations.

In order to distinguish and isolate the WIMP signal, new signatures are of great interest. It was recognized quite early on that the Earth's motion through the galaxy should induce both a seasonal variation in the overall event rate and an overall forward-backward asymmetry in any directional signal [1,2]. The seasonal modulation is problematic, however. Not only is it very small, of the order of $\left(2 v_{\oplus} / v_{\text {halo }}\right)^{2} \approx 0.03-0.05$, but as has become clear as a result of recent claimed dark matter detections, the backgrounds themselves are likely to have seasonal modulation, via modulations in the such things as cosmogenic production of radon, etc.

The directional signature is, a priori, preferable in almost every way. The forward backward asymmetry can be large, $O\left(v_{\odot} / v_{\text {halo }}\right) \approx 1$, and backgrounds are unlikely to reproduce this signature. Nevertheless, in spite of its intrinsic interest and probably because detectors with angular sensitivity are not yet on line, no systematic study of possible angular signals has yet been performed. But because of the difficulty of building such detectors, it is worthwhile to examine in advance what might be possible to learn with directional sensitivity.

Indeed, one of the virtues of this approach is also one of its drawbacks. Angular resolution would provide sensitivity to the detailed features of the galactic halo WIMP distribution. However, because we do not know the nature of this distribution in advance, we cannot assume its form in advance in order to search for a signal. In fact, one might imagine that existing uncertainties are so great that one might not be able to obtain any unambiguous limits on the basis of any lack of asymmetry in the observed signals. It is clear that the estimates which have previously been carried out, for a spherically symmetric isothermal halo, are not sufficient to determine in general how many events may be needed to distinguish signals from backgrounds. In the past decade we have learned that the galaxy has large asphericities. It has also has been argued that the halo dark matter distribution may not resemble an isothermal gas at all [3].

In order to address these features, we have developed a new formalism which allows one to calculate the full differential event rate in detectors, as a function of both energy and angle, for any incident WIMP distribution, without assumptions of spherical symmetry or even cylindrical symmetry. In addition, the full motion of the earth around the Sun is taken into account, not merely the component of the Earth's velocity tangential to the Sun's galactic velocity. While the perpendicular component is largely irrelvent for estimating angular dependent effects if the incident halo is spherically symmetric, this is not the case if this assumption is relaxed. We report here on our general results, leaving a detailed derivation to a later publication. In addition, we summarize the main results from our first application of this formalism. We have generated Monte Carlo distributions for the full spectrum of reported halo models, in order to perform a statistical analysis to determine to what extent any directional signal might unambiguously be distinguishable from an isotropric background. Our results are strking. We find that, independent of the halo model, for energy thresholds and quenching appropriate to the present generation of WIMP detectors, if fine scale angular resolution is possible, fewer than 25 events will be needed to distinguish the halo signal from a uniform background if the signal to noise ratio is large, and fewer than 50 
events would be required if the signal to noise ratio is unity. On the other hand, if only a forward-backward asymmetry is discernable, then between 500-3000 events may be required to distinguish a signal, depending upon the actual halo distribution. In future work we will explore how one might distinguish between different halo models, and how varying angular and energy resolution and WIMP cross sections will effect these estimates.

Consider a WIMP of mass $m_{\chi}$ with its direction specified by the two angles $(\alpha, \beta)$ incident on a fixed nucleus. The WIMP elastically scatters off the fixed nucleus and the scattering event can be described by the two angles $\left(\theta^{*}, \xi\right)$ defined in the center-of-mass frame. The final angle set $(\gamma, \phi)$ describes the direction of the recoiled nucleus in the lab. Note that only the direction of the recoiled nucleus is visible to the detector. Neither the incident nor the scattered direction of WIMP can be observed. The probability of an incident direction $(\alpha, \beta)$ is given by the halo model which defines the WIMP distribution function. Once we relate the three set of angles discussed above using kinematics, we have only to find the proper Jacobian transformations in order to present the final event rate as a function of the visible angles $(\gamma, \phi)$. This is simplified by the introduction of a single function $J(\alpha, \beta ; \gamma, \phi)$ defined as a scalar product of two unit vectors on the sphere,

$$
J(\alpha, \beta ; \gamma, \phi)=[\cos \gamma \cos \alpha+\sin \gamma \sin \alpha \cos (\phi-\beta)] .
$$

This observation leads to the general angle dependent event rates $d R / d \Omega$ and $d^{2} R / d Q d \Omega$ for arbitrary distribution functions. Here $Q(v, J)$ is the energy transferred to the nucleus during the collision with the WIMP and the $\Omega$ is the angle along which the target nucleus recoils. For this work we focus on the angular distribution

$$
\begin{aligned}
& \frac{d R}{d \Omega_{\gamma, \phi}}=\frac{\sigma_{o} \rho_{\chi}}{\pi m_{n} m_{\chi}} \times \\
& \int_{v_{\min }}^{v_{\mathrm{esc}}} v^{3} d v F^{2}(Q) \int d \Omega_{\alpha, \beta} f(v, \alpha, \beta) J(\alpha, \beta ; \gamma, \phi) \Theta(J)
\end{aligned}
$$

where $v_{\min }$ is related to the threshold energy, $\sigma_{0}$ is the low energy WIMP-nucleus scattering cross section, $v_{\text {esc }}=650 \mathrm{~km} / \mathrm{s}$ is the escape velocity of the galaxy, $\rho_{\chi}$ is the local dark matter halo density, $\Theta(J)$ is a unit step function, $m_{n}$ is the mass of the target nucleus, and $F(Q)$ a the form factor suppression for scalar interactions when the WIMP mass couples to the quantum numbers of the entire target nucleus. In this work, we take a simple exponential form for this form factor $F^{2}(Q) \propto \exp \left(-Q / Q_{0}\right)$, where $Q_{0}=\frac{3}{2 m_{n} R_{0}^{2}}$ and $m_{n}$ is the mass of the target nucleus and $R_{0}=0.3+0.91 \sqrt[3]{m_{n}}$ is the radius of the nucleus (in femtometers when $m_{n}$ is in $\mathrm{GeV}$ ).

This formulation of the differential cross section is general enough to accomodate any incident WIMP distribution function $f(v, \alpha, \beta)$. However, when we evaluate these event rates, extra care is needed in the lower limit of the velocity integration defined by the threshold energy of the detector. Unlike the angle independent analysis where the lower limit is fixed once we have the specific threshold or the expected transfered energy, the limit here varies as the incident direction changes for a fixed detector position. This is the main source of the variation of the event rate as a function of angle in the angle dependent spectrum. This change is also taken care of with a division of the lower limit by the same Jacobian (11). The angular distributions shown have been averaged in 5 day bins over the Earth's motion for one year.

We next turn to the galactic halo models. Our desire here is to span the range of realistic possibilities that have been explored in the literature, in order to get a good idea of the existing uncertainties. This might be an important consideration for experimentalists who may devote considerable time to attempting to features in the WIMP distribution that might not be guaranteed to exist. We consider the following: isothermal models, "Evans" models [4] which are axisymmetric and allow for flattening. (previously been studied in the context of annular modulations [5]), co-rotating models with a net angular momentum for the halo [7], and finally the most exteme departure from an isothermal model, using phase space flows dominated by infall into the galaxy for non-dissipative WIMPS, the "Caustic" model [3].

For the isothermal model we will consider three velocity dispersions, $\left\langle v^{2}\right\rangle=3 v_{0}^{2} / 2$ where $v_{0}=150 \mathrm{~km} / \mathrm{s}$, $220 \mathrm{~km} / \mathrm{s}$, and $300 \mathrm{~km} / \mathrm{s}$. For the Evans model the important parameter is the flattening, $q$. We consider the values $q=1$ (cored isothermal distribution), 0.85 , and $1 / \sqrt{2}$ (maximal flattening). For the Caustic model the parameters for the dark matter streams are given in table [1] [6]. If we assume the local density of dark matter is $0.52 \mathrm{GeV} \mathrm{cm}^{-3}$, appropriate to fits to the galactic rotation curve in this model then by summing the density in each caustic peak we find that $60 \%$ of the local density is due to caustics. The other $40 \%$ we assume comes from an isothermal distribution with $v_{0}=220 \mathrm{~km} / \mathrm{s}$.

To model a rotating halo we follow a standard prescription [7]. Let $f_{+}(v, \alpha, \beta)=f(v, \alpha, \beta)$ when $0 \leq \alpha \leq \pi / 2$ and 0 otherwise. Similarly let $f_{-}(v, \alpha, \beta)=f(v, \alpha, \beta)$ when $\pi / 2 \leq \alpha \leq \pi$ and 0 otherwise. The distribution for a rotating halo is then given by $\tilde{f}=(1+\kappa) f_{+}+(1-\kappa) f_{-}$. Note that $\left\langle v^{2} \tilde{f}\right\rangle=\left\langle v^{2} f\right\rangle$. The average velocity of the halo matches the velocity of the Sun when $\kappa=\sqrt{\pi} / 2$.

With these analytic distribution functions, we can utilize our general formalism to calculate the angular event rates, forward backward asymmetries, and annual modulations expected in detectors. A central issue mentioned above, and the one we focus on here, is whether existing uncertainties in galactic halo models might invalidate the approach of utilizing angular resolution to extract a WIMP signature from an otherwise isotropic background. 
To display event rate distributions we choose a set of detector parameters. We use germanium $\left(m_{\mathrm{Ge}}=73 \mathrm{GeV}\right)$ as the target nucleus. Note that germanium has a quenching factor of 0.25 , thus only $25 \%$ of the incident energy gets transfered to the recoiling nucleus. This is important for determining detector thresholds. In the figures an assumed WIMP mass of $60 \mathrm{GeV}$ was used, and thresholds incorporate this quenching factor. We did not assume a specific particle cross section, detector size nor efficiency. Instead we calculate the number of events required for identification of a signal. Based on this number and the local halo density the detector size and efficiency can be determined for a given cross section.

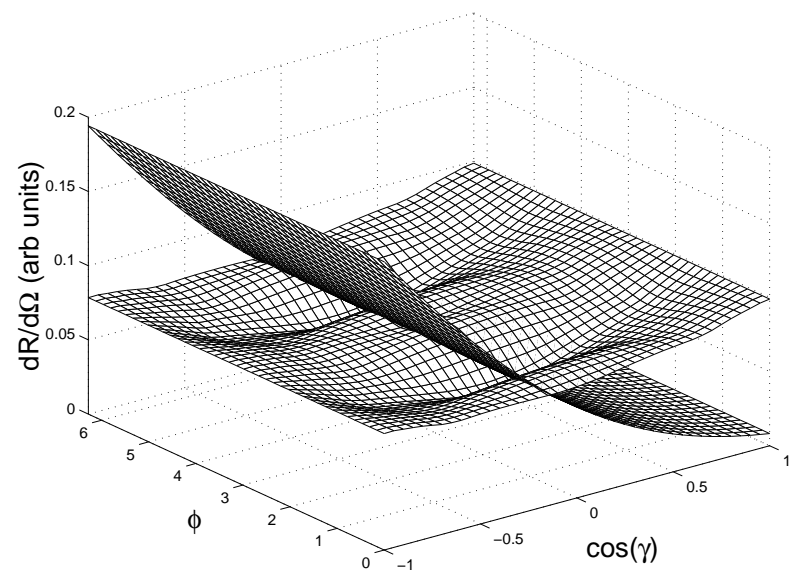

FIG. 1. Angular event rate distribution for an isothermal model with $v_{0}=220 \mathrm{~km} / \mathrm{s}$, and for a caustic infall model with parameters described in the text. The isothermal model falls off exponentially in the forward direction while the caustic model peaks slightly in the forward direction but is more isotropic due to the presence of both caustic and isothermal components in the model.

We employ a maximum likelihood analysis, along with Monte Carlo generation of sample scattering distributions, as follows. We define a likelihood function

$$
\mathcal{L} \equiv \prod_{i=1}^{N_{e}} P\left(\gamma_{i}, \phi_{i}\right)
$$

where $N_{e}$ is the total number of events and $P\left(\gamma_{i}, \phi_{i}\right)$ is the probability of a nuclear recoil in the $\gamma_{i}, \phi_{i}$ direction based on a particular model (e.g. an isothermal distribution). At the $95 \%$ confidence limit when $\log \mathcal{L}_{d R / d \Omega}-\log \mathcal{L}_{\text {flat }}<1$ the two distributions are indistinguishable. We generate 10,000 data sets for each $N_{e}$ and demand that the log-likelihood condition is satisfied less than $5 \%$ of the time. The smallest $N_{e}$ for which this occurs is the minimum number of events required to get a $95 \%$ detection $95 \%$ of the time.

With the above procedure we can determine the number of events required to distinguish the signal from a flat distribution, both for a pure signal and when the signalto-noise ratio, $\mathrm{S} / \mathrm{N}$, is one. For $\mathrm{S} / \mathrm{N}=1$ we replace $P$ with $P_{\text {total }}=\lambda P_{\text {flat }}+(1-\lambda) P_{d R / d \Omega}$ where $\lambda=\frac{1}{1+\mathrm{S} / \mathrm{N}}$. We can perform a similar analysis for the forward-tobackward asymmetry. In this case the probability function $P$ is given by a binomial distribution.

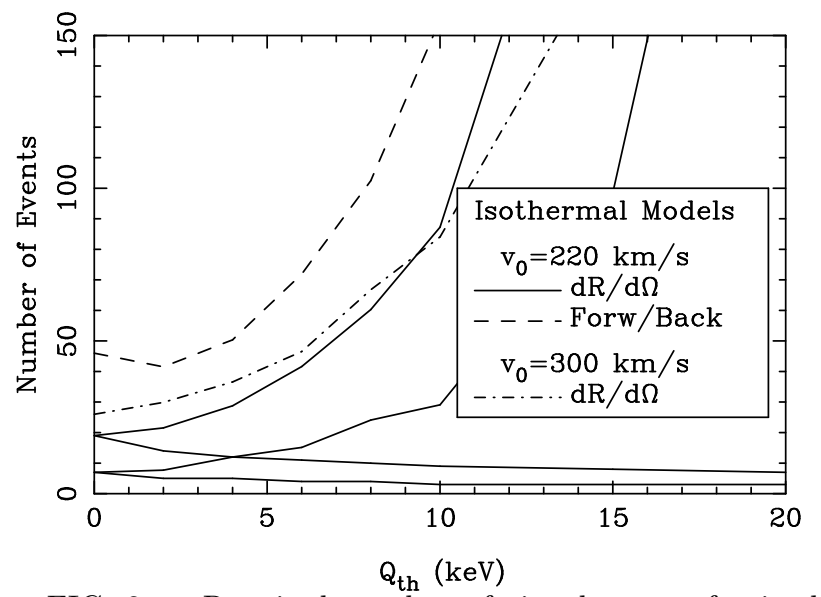

FIG. 2. Required number of signal events for isothermal halos against the null hypothesis. Solid lines correspond to the $v_{0}=220 \mathrm{~km} / \mathrm{s}$ and dashed lines to $v_{0}=300 \mathrm{~km} / \mathrm{s}$ - For the former, both $\mathrm{S} / \mathrm{N}=1$ case (upper) and no noise cases are shown as are both the number of signal events as a function of threshold and as the equivalent number of events required for zero threshold. For the other case only the latter curve, for $\mathrm{S} / \mathrm{N}=1$ is shown. This latter curve is also displayed when only the forward-backward asymmetry is used to probe a $v_{0}=220 \mathrm{~km} / \mathrm{s}$ isothermal halo.

The results as a function of the threshold for an isothermal halo with velocity dispersions of $220 \mathrm{~km} / \mathrm{s}$ and $300 \mathrm{~km} / \mathrm{s}$ are given in Figure 2 and for a forwardbackward asymmetry measurement for the $220 \mathrm{~km} / \mathrm{s}$ halo. In this figure, for the $220 \mathrm{~km} / \mathrm{s}$ halo case, the bottom sets of lines give the number of signal events required as a function of the threshold. Fewer events are required to distinguish a halo from a flat distribution in this case at higher threshold because the anisotropy of the distribution becomes more dramatic as higher scattering energies, and thus higher incident velocities relative to the Earth, are required. However, while fewer events are required as the threshold is increased, even fewer events are expected, for a given detector size, as the threshold is increased. Thus, one does not win by simply raising the theshold. To make this clear, the upper curves show the number of events (proportional to the size of the detector) that would be needed to be observed in a zero threshold detector in order to obtain the requisite number of events at each non-zero threshold. This curve is a monotonically increasing function of threshold. Clearly, the lower the threshold, then, the smaller the detector, and the smaller the total number of scatterings required 
in the detector in order to distinguish the distribution from a flat distribution.

The upper two sets of curves for the $220 \mathrm{~km} / \mathrm{s}$ halo case in the figure represent the same requirement, but now in the case when the signal to noise ratio, $\mathrm{S} / \mathrm{N}=1$ at each value of the threshold. The number of events plotted are the signal events. The noise events would lead to twice as many total events actually being detected. For the $300 \mathrm{~km} / \mathrm{s}$ case, the forward-backward asymmetry, and the the co-rotating halo, Caustic, and Evans models (fig. 3 ) only the latter $\mathrm{S} / \mathrm{N}=1$, zero threshold-equivalent number of events are shown.

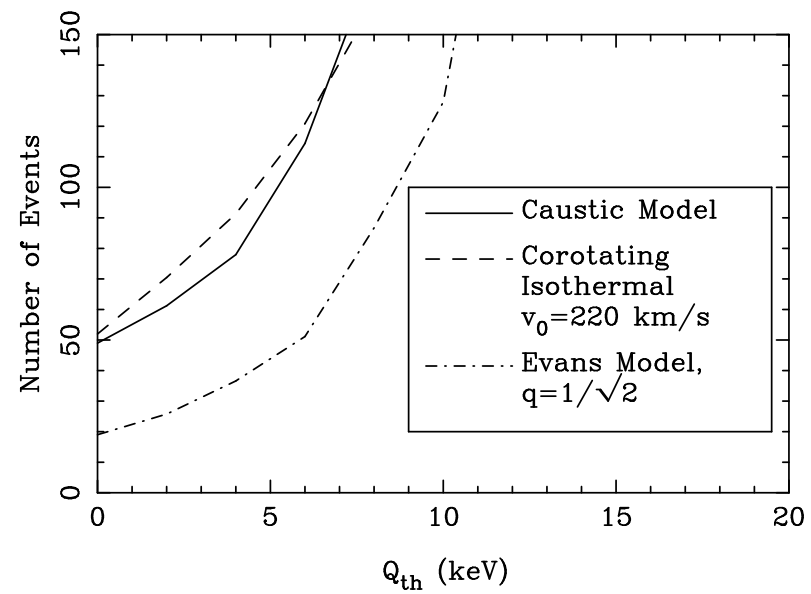

FIG. 3. Required number of signal events for the Caustic model, a co-rotating, and an Evans model (with $q=1 / \sqrt{2}$ ) against the null hypothesis, as in figure 2 . Only $\mathrm{S} / \mathrm{N}=1$, zero threshold-equivalent number of events are shown for these models.

Perhaps the most suprising result of our analysis is that, independent of the existing uncertainties in the halo distribution, that less than 50 detected events for a signal to noise ratio of unity would be required in order to unambiguously distinguish a halo induced signal from that due to a flat background, assuming fine grained angular resolution. If, however, only forward/backward sensitivity were available, the requisite number of events increases dramatically for non-isothermal distributions. This number rises to 500 for the co-rotating case, and 3000 for the Caustic case. Next, we note the suprising result that the Caustic distribution, which in principle is the most distinctive halo distribution of all, requires the greatest number of events in order to be distinguished from a flat distribution. There are two reason for this. First, because this distribution is dominated by infall components, the induced anisotropy in the pure cautic distribution is in the opposite direction to that predicted in a standard isothermal model. Adding a isothermal component then tends to cancel the anisotropy induced by the Caustic flows. In addition, because there is no exponential tail for the caustic, the aniostropy tends to rise linearly in the forward instead of exponentially (in the backward direction) as in the pure isothermal models.

For the same reason it is clear that the Caustic distribution should be more easily distinguishable from these other distributions than it is from a flat background. The formalism we have presented here will allow quantitative estimates for any observables to be derived for any incident halo distribution. Indeed, in future work we plan to investigate how many events will be required in detectors in order to distinguish between the various halo models, as well as incorporating additional detector features such angular resolution and energy detection uncertainties as well as the theoretical distinctions between spin-dependent and spin-independent scattering cross sections. Our initial results, however are encouraging. Independent of halo uncertainties, good angular resolution would allow an anisotropic halo signal to be differentiated from a flat background signal with a fraction of the number of events that would be needed to probe for annual modulation, or other expected spectral features due to a WIMP halo in the galaxy. If a forward-backward asymmetry is all that can be detected, the number of events required is model dependent, and can be quite large, however.

This work was supported by the DOE.

[1] B. Sadoulet, unpublished

[2] D.N. Spergel, Phys. Rev. D37, 1353 (1988).

[3] P. Sikivie, I.I. Tkachev, and Y. Wang, Phys. Rev. D56, 1863 (1997).

[4] N.W. Evans, Mon. Not. R. Astron. Soc. 260, 191 (1993).

[5] M. Kamionkowski and A. Kinkhabwala, Phys. Rev. D57, 3256 (1998).

[6] P. Sikivie, private communication.

[7] D. Lynden-Bell, MNRAS 136, 101 (1967).

TABLE I. Velocity flows of dark matter from the Caustic model (for $h=0.75, \epsilon=0.28, j_{\max }=0.25$ Sikivie model). Velocities are given in the rest frame of the galaxy.

\begin{tabular}{ccccc}
\hline \hline Flow & $\rho^{\mathrm{a}}$ & $v_{x}^{\mathrm{b}}$ & $v_{y}{ }^{\mathrm{b}}$ & $v_{z}^{\mathrm{b}}$ \\
\hline 1 & 0.4 & 140 & 0 & \pm 600 \\
2 & 0.9 & 250 & 0 & \pm 500 \\
3 & 2.0 & 350 & 0 & \pm 395 \\
4 & 6.1 & 440 & 0 & \pm 240 \\
5 & 9.6 & 440 & \pm 190 & 0 \\
6 & 3.0 & 355 & \pm 290 & 0 \\
7 & 1.9 & 295 & \pm 330 & 0 \\
8 & 1.4 & 250 & \pm 350 & 0 \\
9 & 1.0 & 215 & \pm 355 & 0 \\
10 & 1.1 & 190 & \pm 355 & 0 \\
\hline \hline
\end{tabular}

${ }^{\mathrm{a}}$ In units of $10^{-26} \mathrm{~g} \mathrm{~cm}^{-3}$

${ }^{\mathrm{b}}$ velocities shown are in $\mathrm{km} \mathrm{s}^{-1}$ 